\title{
Hacia un modelo educativo para el emprendimiento ${ }^{1}$
}

DOI: https://doi.org/10.21158/01208160.n87.2019.2420

Fecha de recepción: 18 de septiembre de 2018 Fecha de aprobación: 02 de junio de 2019
Ulises Segura-Barón ${ }^{2}$ Universidad Piloto de Colombia ulises-segura@unipiloto.edu.co

Henry Alberto Novoa-Matallana ${ }^{3}$ Universidad Piloto de Colombia hmn1212@gmail.com

Maria Del Cielo Burbano-Pedraza ${ }^{4}$ Universidad Piloto de Colombia maria-burbano@unipiloto.edu.co

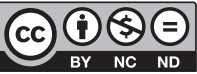

Cómo citar este artículo / To reference this article / Comment citer cet article / Para citar este artigo:

Segura-Barón, U.; Novoa-Matallana, H. A.; Burbano-Pedraza, M. (2019). Hacia un modelo educativo para el emprendimiento. Revista Escuela de Administración de Negocios, (87), 173-191. DOI: https://doi.org/10.21158/01208160.n87.2019.2420

\section{Resumen}

Con base en las consideraciones teóricas y conceptuales, la visión desde la investigación en emprendimiento, la revisión de las condiciones institucionales y el análisis de relaciones entre variables en este tema, se elaboró la propuesta de un modelo piloto de emprendimiento dinámico e innovador el cual se presenta a partir de un eje articulador denominado Centro de Emprendimiento para la Universidad Piloto de Colombia, seccional Alto Magdalena.

El enfoque metodológico da cuenta de triangulación de información cuantitativa y cualitativa, que inició con una revisión documental sobre la normatividad vigente acerca del potencial que la educación y la investigación tienen para el emprendimiento, así como su articulación con la estructura determinada por el Estado y las necesidades existentes en lo relativo a transferencia del conocimiento desde la universidad al sector productivo y social y prosiguió con el desarrollo de procesos de discusión institucional, contrastando la información recabada con las percepciones de los actores del proceso.

${ }^{1}$ El presente artículo es un producto del proyecto de investigación "Hacia un proyecto educativo para el emprendimiento", realizado en la Universidad Piloto de Colombia, en el 2017, con la finalidad de generar una propuesta para la operación del modelo de emprendimiento denominado "Emprendimiento dinámico innovador".

${ }^{2}$ Doctor en Educación, Universidad Norbert Wiener Perú; Magister en desarrollo educativo y social, Universidad Pedagógica Nacional. CINDE. Economista, Universidad Cooperativa de Colombia. Especialista en Gerencia de proyectos, Universidad Piloto de Colombia; Especialista en educación ambiental, Universidad del Tolima; Especialista en innovación y pedagogía universitaria, Universidad Piloto de Colombia. ORCID: https://orcid.org/0000-0003-4879-4957

3 Tecnólogo en Administración de Empresas de Economía Solidaria, Universidad Santo Tomas - Bogotá. Contador Público, Universidad Piloto de Colombia - Girardot. Especialización en Revisoría Fiscal, Universidad Piloto de Colombia - Girardot. Especialización en Gerencia, Universidad Ceipa - Medellín. Maestría en Gerencia de Negocios, Escuela de Administración de Empresas Barcelona - España. ORCID: https://orcid.org/0000-0003-1976-2219

${ }^{4}$ Licenciada en español e inglés, Universidad Pedagógica Nacional; Magíster en Literatura Hispanoamericana, Instituto Caro y Cuervo, Seminario Andrés Bello. ORCID: https://orcid.org/0000-0001-7444-1536 
En este marco se determinaron las variables que compusieron la línea de investigación, las cuales fueron: el emprendedor, el entorno y las relaciones existentes entre el emprendedor y el entorno por las acciones de los actos de emprendimiento.

Así las cosas, se concluye que resulta evidente la importancia de fortalecer la investigación en emprendimiento mejorando sus niveles cuantitativos y cualitativos y definiendo un derrotero o argumentación real y significativa para soportar todo tipo de políticas y proyectos en apoyo al tema, sobre la base de la triada fundamental de desarrollo: universidad, empresa y Estado.

Palabras clave: emprendimiento; modelo de emprendimiento, emprendimiento universitario; apoyo al emprendimiento; potencial en emprendimiento; acciones de emprendimiento; incubadoras de emprendimiento.

\title{
Towards an educational model for entrepreneurship
}

\begin{abstract}
Based on the theoretical and conceptual considerations, the vision from the research in entrepreneurship, the review of institutional conditions and the analysis of relationships between variables in this topic, the proposal was developed a pilot model of dynamic and innovative entrepreneurship which is presented based on an articulating axis called the Entrepreneurship Center for the Pilot University of Colombia, Alto Magdalena section.

The methodological approach accounts for triangulation of quantitative and qualitative information, which began with a documentary review of current regulations on the potential that education and research have for entrepreneurship, as well as its articulation with the structure determined by the State and existing needs regarding the transfer of knowledge from the university to the productive and social sector and continued with the development of institutional discussion processes, contrasting the information gathered with the perceptions of the process actors.

In this framework, the variables that made up the line of research were determined, which were: the entrepreneur, the environment and the relationships between the entrepreneur and the environment through the actions of the entrepreneurial acts.

Thus, it is concluded that the importance of strengthening entrepreneurship research is evident by improving its quantitative and qualitative levels and defining a real and meaningful course or argument to support all kinds of policies and projects in support of the subject, based on the Fundamental development triad: university, business, and State.
\end{abstract}

Keywords: entrepreneurship; entrepreneurship model; university entrepreneurship; entrepreneurship support; entrepreneurship potential; entrepreneurship actions; entrepreneurship incubators.

$\longrightarrow$ Rumo a um modelo educativo para o empreendedorismo

\section{Resumo}

Com base nas considerações teóricas e conceituais, na visão da pesquisa em empreendedorismo, na revisão das condições institucionais e na análise das relações entre as variáveis deste tópico, se elaborou a proposta de um modelo piloto de empreendedorismo dinâmico e inovador, que é apresentado baseado em um eixo articulador chamado Centro de Empreendedorismo da Universidade Piloto da Colômbia, seção Alto Magdalena. 
A abordagem metodológica explica a triangulação de informações quantitativas e qualitativas, iniciada com uma revisão documental das regulamentações atuais sobre o potencial que a educação e a pesquisa têm para o empreendedorismo, bem como sua articulação com a estrutura determinada pelo Estado e necessidades existentes em relação à transferência de conhecimento da universidade para o setor produtivo e social e continuou com o desenvolvimento de processos de discussão institucional, contrastando as informações coletadas com as percepções dos atores do processo.

Nesse marco, foram determinadas as variáveis que formavam a linha de pesquisa: empreendedor, o entorno e relações entre empreendedor e entorno, por meio das ações dos atos empreendedores.

Assim, conclui-se que a importância de fortalecer a pesquisa sobre empreendedorismo é evidente, melhorando seus níveis quantitativos e qualitativos e definindo um curso ou argumento real e significativo para apoiar todos os tipos de políticas e projetos de apoio ao assunto, com base na tríade do desenvolvimento fundamental: universidade, empresa e Estado.

Palavras-chave: empreendedorismo; modelo de empreendedorismo; empreendedorismo universitário; apoio ao empreendedorismo; potencial de empreendedorismo; ações de empreendedorismo; incubadoras de empreendedorismo.

\section{Vers un modèle éducatif orienté à l'entrepreneuriat}

\section{Résumé}

Cet article présente un modèle d'entrepreneuriat innovant mis en place par le Centre pour l'Entrepreneuriat de l’Université Pilote de Colombie, section Haut Magdalena.

Ce modèle entrepreneurial innovant et dynamique se base sur plusieurs principes fondamentaux tels que les considérations théoriques et conceptuelles, une vision issue de la recherche sur l'entrepreneuriat, la révision des conditions institutionnelles et l'analyse des relations entre les variables afférentes à ce thème.

L’approche méthodologique prend en compte la triangulation des informations quantitatives et qualitatives et commence par une analyse documentaire de la réglementation en vigueur sur le potentiel offert par l'éducation et la recherche sur l'esprit d'entreprise et son articulation avec la structure étatique et gouvernementale des besoins existants en matière de transfert de connaissances du secteur éducatif vers le secteur productif, et à la mise en place de processus de discussion institutionnelle contrastant les informations recueillies avec les perceptions des différents acteurs.

Les variables déterminantes composant cette ligne d'investigation sont les suivantes : l'entrepreneur, l'environnement, les relations entre l'entrepreneur et l'environnement, les actions de l'entreprise.

Larticle conclut sur l'importance du renforcement qualitatif et quantitatif de la recherche en entrepreneuriat, sur la nécessité de définir un processus réel et significatif d’appui aux politiques et projets en faveur de l'entrepreneuriat sur la base d'un triptyque pour le développement fondamental : université, entreprise, état.

Mots-clés: entrepreneuriat; modèle d'entreprise, esprit d'entreprise dans les universités; soutien à l'entrepreneuriat; potentiel entrepreneurial; actions entrepreneuriales; incubateurs en entrepreneuriat. 


\section{Introducción}

$\mathrm{R}$ esultado de la investigación Hacia un proyecto educativo para el emprendimiento y a propósito de la importancia del tema para el desarrollo social y económico del país, se presenta este ejercicio de acercamiento y análisis desde una propuesta educativa dirigida al emprendimiento en la Seccional del Alto Magdalena de la Universidad Piloto de Colombia, la cual, a través del desarrollo de la investigación y mediante revisiones teóricas conceptuales y el desarrollo de procesos de discusión institucional con los actores, construye una alternativa válida y pertinente para los escenarios educativos del país, con lo que se cumple una finalidad específica del proceso investigativo: aportar al diálogo de saberes desde el ejercicio académico en el marco del emprendimiento.

En términos generales, es fundamental retomar la Ley 1014 de 2006, «De fomento a la cultura del emprendimiento», cuyo artículo 1 plantea el marco general y conceptual de este fenómeno económico, cultural y social: actores, aspectos y relaciones tales como cultura, emprendedor, emprendimiento, empresarialidad, planes de negocio y, finalmente, el proceso educativo como tal (Ley 1014 de 2006, p. 1).

Con esto se crean condiciones para una política de Estado orientada hacia la articulación de los sistemas educativo y productivo en el país, de modo que se promueve el emprendimiento y la creación de empresas, así como se fomenta el desarrollo de micro y pequeñas empresas innovadoras y generadoras de valor. De igual forma, se busca, por medio de las diferentes redes, adelantar un proceso efectivo de acompañamiento y sostenibilidad para las empresas que se creen, con lo que se garantiza un entorno propicio y seguro, pero, sobre todo, innovador. De esta manera, en conformidad con la Ley 1014 de 2006 (p. 3), el proceso debe garantizar:

Formación integral en aspectos y valores como
desarrollo del ser humano y su comunidad,
autoestima, autonomía, sentido de pertenencia
a la comunidad, trabajo en equipo, solidaridad,
asociatividadydesarrollo del gusto por la innovación
y estímulo a la investigación y aprendizaje
permanente.

En este orden de ideas, la formación para el emprendimiento que se plantea desde el Estado colombiano se concreta en la formación integral de personas productivas, de manera que se garantizan así procesos continuos y de permanente desarrollo en las competencias en las que el emprendedor necesita crecer: competencias básicas, competencias laborales, competencias ciudadanas y competencias empresariales. 


\section{La dimensión metodológica}

$\mathrm{P}$ ara el desarrollo de la investigación se aplicó una metodología de carácter mixto, por la cual se determinó como actores principales a participantes del acto educativo tales como los estudiantes, los profesores, los egresados y, por supuesto, las unidades de apoyo de la gestión académica.

El enfoque metodológico da cuenta de la triangulación de información cuantitativa y cualitativa. Para esto se inicia con una revisión documental sobre la normatividad vigente acerca del potencial que la educación y la investigación tienen para el emprendimiento, así como su articulación con la estructura determinada por el Estado y sus necesidades en cuanto a transferencia del conocimiento desde la universidad al sector productivo y social, información que se contrasta con las percepciones que provienen de los actores del proceso.

Así las cosas, el documento se construyó desde: a) la indagación en términos de la normatividad que el gobierno nacional estableció para la educación; b) la articulación con el ámbito universitario y sus implicaciones, en particular con relación a la institucionalidad para el caso de la Universidad Piloto de Colombia; c) una revisión general en torno al emprendimiento desde la órbita de las funciones sustantivas de la universidad; d) un diagnóstico al interior de la universidad que se soporta en los procesos adelantados desde la vicerrectoría académica con las decanaturas de los programas académicos; e) la visión que tienen los estudiantes de los procesos para el emprendimiento y su incidencia en la percepción sobre su formación académica y profesional; f) la presentación de la propuesta de modelo educativo para la seccional; yg) las correspondientes conclusiones del proceso adelantado.

La propuesta presenta una triada en su fundamentación. En primer lugar, desdelos ejercicios realizados por la rectoría, la vicerrectoría y proyección social institucional. Luego, desde el emprendimiento o entrepreneurship como un espacio del conocimiento aún en proceso de consolidación, en el marco de la investigación y sus componentes conceptuales y al concretar el proceso con el diagnóstico interno en la seccional del Alto Magdalena.

Se realizó un grupo focal con los siete decanos y el vicerrector académico de la seccional con preguntas ordenadoras del tema acerca del conocimiento y la experiencia temática, el estado actual, los soportes y las diversas opciones, como, por ejemplo, los trabajos de grado y las prácticas, así como sus posibilidades en el marco de los registros calificados y la acreditación institucional.

Por otra parte, se aplicó la encuesta de percepción a los estudiantes de la seccional de los semestres VII, VIII y IX, en su totalidad, pues se considera que son los de mayor posibilidad de formación en razón a su estado de avance en sus planes de estudio. En este sentido, se indagó acerca de: identificación del concepto, experiencia en el tema, interés en el tema, posibilidad de opción de grado y el interés y necesidad de formación para el emprendimiento. 


\section{Componentes desde la reflexión teórica}

Ce inicia el proceso de revisión de la literatura en torno a la reflexión educativa para el emprendimiento desde lo expuesto por Osorio y Pereira (2011), quienes ofrecen una mirada desde la teoría social cognitiva, en la búsqueda de detectar las capacidades necesarias que permitan tomar decisiones emprendedoras.

El aporte fundamental de este estudio es el planteamiento del siguiente interrogante: ¿Cuál es el potencial de la educación para el emprendimiento? Así, se encontró que, desde la teoría social cognitiva, se visualiza un marco de referencia que delimita y configura, a partir del cual se construyen de forma acertada las variables que especifican su dominio. Se trata, entonces, fundamentalmente, de desarrollar las capacidades o las competencias básicas para hacer que las personas tomen decisiones emprendedoras.

Osorio y Pereira (2011, p.29), en esencia, concluyen:

Para educar para el emprendimiento no es suficiente entender los principios y prácticas de los negocios, y conseguir habilidades, atributos y actitudes más allá de lo comercial. La educación, como elemento complementario del aprendizaje, debe ayudar a que al individuo observe su conducta, a través de mecanismos sociales y cognitivos, para el emprendimiento.

Por otra parte, en torno a la relación de la investigación, el emprendimiento y la universidad, Chavarro y Orozco (2008) sostienen la posibilidad de potenciar tanto la investigación como la educación, en la medida en que los conocimientos se llevan a la práctica y, a través de esta, se genera valor social.

De esta forma, la universidad media para articular el conocimiento científico, la docencia y la labor social, pues en esta acción se concretan los nuevos conocimientos. De ahí la discusión sobre cómo la universidad logra activar su participación en el desarrollo económico y social.
Los autores abordan el tema bajo una pregunta orientadora y general: ¿Cuál es el rol institucional de la universidad frente al emprendimiento y la innovación para el beneficio de la sociedad? A partir de este interrogante se da apertura al debate que hoy sigue abierto, pero del que se plantean algunos aspectos orientadores. En primer lugar, si bien es cierto que la Ley 1014 propende al fomento a la cultura emprendedora, en particular se centra en los colegios de primaria y la educación media a través de la cátedra transversal de emprendimiento, lo que sitúa muy bien el origen del proceso desde la educación en los niños y los jóvenes; sin embargo, es necesario establecer un vínculo muy claro entre los sistemas educativo y productivo colombianos, por lo que, en especial la universidad — la cual, más allá de educar, investiga y genera conocimientos que, evidentemente, se requiere apropie todo el sistema productivo- genera oportunidades de innovación y emprendimiento.

Ahora bien, es claro y contundente lo que expresan los autores: «Si hay un punto en el que podemos apreciar claramente la utilidad y visibilidad de las actividades de investigación, ese es el impacto en la generación de nuevas empresas que posibiliten la creación de valor económico y valor social» (Chavarro y Orozco 2008, p. 67).

Las redes de relaciones sociales se interpretan como mecanismos de éxito en la acción de emprendimiento a raíz del valor del capital social que allí se genera.

Se asumen dos tipos de enfoques. Uno desde la empresa innovadora, con un ambiente propicio para la innovación, y el otro desde los intereses de los emprendedores por entrar al mundo de los negocios. En estos enfoques se recogen aspectos psicológicos, la motivación, la autoimagen, la autoestima, el estatus social ante la creación de empresas en general, así como factores cognitivos y educativos para el desarrollo desde la perspectiva educativa científico y tecnológico-. 
Por otra parte, Orozco y Chavarro afirman:

En el libro del Banco Mundial (Garnsey, 2007) sugiere que no siempre el emprendimiento de las universidades es recibido favorablemente por las organizaciones que controlan ciertos mercados y negocios, por lo que se debe analizar el modelo ante la posibilidad de apoyo de grupos de negocios que sustenten esta posibilidad. (2008, p. 83)

Esto significa que se abre la posibilidad inminente que plantea entre una universidad de investigaciones o una de emprendimiento. Sin que esto conduzco a un antagonismo, es necesario revisar los intereses que se encuentran en el escenario dispuesto.

A propósito de una gran conclusión, los autores señalan:

Consideramos que las universidades deben pensar la diferencia entre auspiciar el emprendimiento por necesidad o por oportunidad, el primero enfocado a promover un entusiasmo empresarial como estrategia de autoempleo, pero sin una base de conocimiento que brinde garantías de apoyo en la competitividad de largo plazo, y el segundo que aprovecha la invención y genera la innovación como base de la competitividad. (2008, p. 89)

Así, entonces, el emprendimiento originado en la universidad debe entenderse mucho más allá de una cultura empresarial o de cursos de emprendimiento; en concreto, en la fuente de planes de negocios y nuevas empresas sostenibles. Esto es coherente con la noción de empresario emprendedor desde la universidad:

[... el emprendimiento de la universidad adquiere sentido, en virtud de que el emprendimiento es la creación de nuevas organizaciones que aprovechan las oportunidades de la invención científica y técnica, organizan su trabajo para reducir los riesgos en la coordinación de recursos escasos y salen de la universidad en forma de spin-off o spin-out para afrontar la incertidumbre en los mercados y ejecutar acciones productivas, que para nosotros, ofrecen posibilidades para crear valor social. (2008, p. 70)

En este plano, la propuesta SAM remite a articular la necesidad y la oportunidad del emprendedor desde la generación de empleo y la innovación para el desarrollo empresarial del país.
Ahora bien, al analizar el fenómeno en el contexto de la universidad colombiana y de conformidad con lo expuesto por Colciencias, en los últimos diez años - sobre todo como respuesta a las exigencias de entes reguladores y a la divulgación del temase produjo un incremento considerable en la cultura emprendedora, por lo que se puede afirmar, en términos generales, que el emprendimiento en cuanto forma de investigación científica y de producción de conocimiento presenta avances significativos.

Así lo refleja un estudio acerca de la investigación para el emprendimiento (Matiz, 2009), la cual remite al caso colombiano con base en el sistema de información Scienti de Colciencias y toma como muestra 1240 artículos publicados en journals de nivel internacional que versan sobre una variedad de disciplinas académicas, tales como gerencia (396 artículos) con el 31,9\%, economía (268) con 21,6\%, finanzas (140) con el 11,3\%, mercadeo (122) con el $9,8 \%$, y contabilidad (50), con un $4 \%$.

$\mathrm{Al}$ respecto, es pertinente la conclusión del autor:

Investigadores, entidades de educación, sector privado, gobiernos locales, regionales y el gobierno nacional, deben trabajar no sólo en la generación de una cultura que privilegie el emprendimiento como opción de vida y herramienta de desarrollo, sino como un área de construcción de conocimiento. (2009, p. 180)

En este orden de ideas, es preciso señalar algunas miradas al concepto como tal, dado que, en particular, la ciencia económica lo ubica bajo el interrogante «iQué hace?», las ciencias humanas les interesa es el sujeto «iQuién y por qué?», y las organizacionales se preguntan por el proceso.

Así las cosas, el emprendedor piloto genera ideas, construye de forma permanente, aprende — según sus competencias, metas y objetivos-, convierte y vuelve sobre el proceso para seguir innovando. Esta es la base para construir una educación que permita fortalecer sus factores de éxito, identificados en sus variaciones particulares. 
Finalmente, en este sentido y como se cita en Crissien-Castillo (2009), se presenta la tabla 1 de Rodríguez y Jiménez.

Tabla 1. Algunos exponentes del emprendimiento

\begin{tabular}{|c|c|c|c|}
\hline R. CANTILLON & $\begin{array}{l}\text { Es un agente que compra medios de } \\
\text { producción a ciertos precios, los transforma } \\
\text { y combina de forma ordenada, y obtiene de } \\
\text { allí un nuevo producto. El emprendedor, a } \\
\text { diferencia de otros agentes, toma el riesgo, } \\
\text { es decir, no tiene plena certeza, por ende, es } \\
\text { un agente tomador de riesgos que afecta el } \\
\text { comportamiento de un mercado (como se } \\
\text { cita en Thorton, 1986). }\end{array}$ & J. SHUMPETER & $\begin{array}{l}\text { El empresario es el destructor } \\
\text { creativo. Mediante la innovación, } \\
\text { entendida como hacer algo nuevo, } \\
\text { bien sea un producto, un nuevo } \\
\text { proceso o la mejora de uno existente, } \\
\text { se desequilibran los mercados. } \\
\text { El empresario es el motor de la } \\
\text { economía para este autor. }\end{array}$ \\
\hline P. DRUKER & $\begin{array}{l}\text { Plantea al empresario como un innovador. } \\
\text { EL empresario innovador busca el cambio, } \\
\text { responde a él y lo explota como una } \\
\text { oportunidad (Druker 1985). Lo define, } \\
\text { en específico, como el entrepreneur, } \\
\text { el empresario que es innovador, y al } \\
\text { entrepreneurship como el empresariado } \\
\text { innovador. Establece la diferencia entre } \\
\text { un pequeño negocio tradicional y un } \\
\text { verdadero emprendimiento, el cual es } \\
\text { aquel que genera cambios en los mercados } \\
\text { (Druker 2002). No todo negocio es un } \\
\text { emprendimiento innovador. }\end{array}$ & A. SMITH & $\begin{array}{l}\text { De forma breve trata el tema del } \\
\text { entrepreneurship, bajo el término } \\
\text { inglés business management. Este } \\
\text { autor apunta a obtener el máximo } \\
\text { rendimiento de los recursos y } \\
\text { así lograr el equilibrio. Incluye al } \\
\text { empresario en el marco de los factores } \\
\text { externos o fuerzas externas que } \\
\text { afectan la dinámica económica. }\end{array}$ \\
\hline A. MARSHAL & $\begin{array}{l}\text { Introduce el concepto según el cual } \\
\text { los factores de producción no son tres } \\
\text { sino cuatro. A los factores tradicionales } \\
\text { — capital, tierra y trabajo- agrega la } \\
\text { organización, y la define como el factor } \\
\text { coordinador que atrae otros factores y los } \\
\text { agrupa. EL entrepreneurship es el elemento } \\
\text { que está detrás de la organización y la } \\
\text { maneja. Plantea que los emprendedores } \\
\text { tienen como característica básica el } \\
\text { liderazgo y actúan en condiciones de } \\
\text { incertidumbre. Aun cuando comparte } \\
\text { con otros autores que los emprendedores } \\
\text { presentan ciertas habilidades natas, plantea } \\
\text { que estas se pueden aprender y se pueden } \\
\text { adquirir. }\end{array}$ & H.STEVENSON & $\begin{array}{l}\text { Emprender es perseguir la } \\
\text { oportunidad más allá de los } \\
\text { recursos disponibles y controlables } \\
\text { en la actualidad. El empresario es } \\
\text { un creador de empresa y busca } \\
\text { la generación de riqueza. Una } \\
\text { característica del empresario, a fin de } \\
\text { que se determine como emprendedor, } \\
\text { es que sea innovador. Para este autor } \\
\text { la innovación no es solo de producto } \\
\text { o servicio, sino también de procesos } \\
\text { o mercados. Establece la diferencia } \\
\text { entre el empresario exitoso y el } \\
\text { ejecutivo exitoso. }\end{array}$ \\
\hline S. MILL & $\begin{array}{l}\text { Inglés de nacimiento. Lamenta que en el } \\
\text { idioma inglés no existiera una palabra con } \\
\text { el mismo significado de la palabra francesa } \\
\text { entrepreneur. Enfatiza la importancia } \\
\text { del emprendimiento en el crecimiento } \\
\text { económico, y cómo el desarrollo del } \\
\text { entrepreneurship requiere de habilidades } \\
\text { no comunes. }\end{array}$ & M. WEBER & $\begin{array}{l}\text { Este autor plantea la tarea del } \\
\text { emprendedor como una forma de } \\
\text { vida o una profesión de vida. Se } \\
\text { caracteriza por la frugalidad para el } \\
\text { logro de riqueza y, por ende, tiene una } \\
\text { conducta y una ética diferente que } \\
\text { adquiere códigos específicos para el } \\
\text { logro del objetivo. }\end{array}$ \\
\hline
\end{tabular}

Fuente. Rodríguez y Jiménez, como se cita en Crissien-Castillo, 2009. 


\section{Desarrollo}

$\mathrm{E}_{\mathrm{e}}^{\mathrm{se}}$ ste apartado se construyó a partir de un ejercicio de diálogo interno con la comunidad académica en general, en específico: 1) Vicerrectoría académica, 2) programas académicos y decanaturas; y 3) estudiantes. Los resultados se presentan a continuación.

\subsection{Desde vicerrectoría académica}

En la seccional no se cuenta con la estructura mínima para el fomento del emprendimiento. Todo depende del esfuerzo de programas académicos, en específico de Contaduría pública, en los que se asignan algunas horas de dedicación a docentes, por lo que se requiere sustentar los procesos desde la incubación —creación-, y la formalización y la aceleración asesorías y consultorías - de empresas. Se resalta la concepción de la formalización de empresas desde interrogantes fundamentales como, por ejemplo «iqué?», «icómo?»y «ipara qué?». Se concluye que el emprendimiento debe tener una importancia similar a la de la investigación y avenirse con ella, pues desde la investigación se generan empresas, y desde el Centro de Emprendimiento se realiza el acompañamiento necesario.

\subsection{Desde los programas académicos}

\subsubsection{Contaduría pública.}

Tiene una importante trayectoria de apoyo a los procesos de formalización empresarial en mipymes de la comunidad de Cundinamarca, desde los cuales se han ejecutado importantes procesos. En efecto, el programa involucra en su misión la formación integral e íntegra del futuro profesional contador público piloto, así como el pensamiento crítico con un sentido humanizador de las situaciones coyunturales que afronta hoy el mundo, cualidades que, entre otras direcciones, se orientan al desarrollo del emprendimiento.

\subsubsection{Ingeniería financiera.}

Se parte de la acepción del término inglés entrepreneur o pionero, y su vinculación con la teoría económica, según Keynes y Chumpeter, de manera que se convierte el emprendedor en el centro del sistema económico. Considera que el emprendimiento es cuestión de una actitud que puede desarrollar el ser humano desde el mismo momento en el que se enfrenta a una situación que obstaculiza y no posibilita la obtención de una meta, y la forma en que este busca resolverla.

\subsubsection{Ingeniería de sistemas.}

Contempla que el emprendimiento supone las acciones necesarias para llevar a cabo un plan empresarial que permita desarrollar un producto o servicio, que cumpla con las condiciones para comercializarlo. En su visión plantea que sus profesionales serán reconocidos, entre otras características, por ser innovadores en el desarrollo de software y estar comprometidos con el desarrollo social autosostenible.

\subsubsection{Administración logística.}

El emprendimiento es una actitud que incita a la persona a hacer cosas extraordinarias, orientadas a mejorar su entorno a partir de la identificación de problemáticas reales. El programa lo percibe desde las perspectivas de creación de empresa y de innovación de procesos. Se considera que el programa académico requiere mejorar sus estrategias $y$ actividades en este sentido, pero no solo desde la mirada local, sino también nacional y, por qué no, internacional.

\subsubsection{Administración turística.}

El emprendimiento es una iniciativa de cada persona en la que confluyen el entorno, el liderazgo personal y la actitud. Es una característica que, mediante las técnicas y herramientas de formación, se convierte en una opción de vida y un modelo de negocio 
replicable. En la actualidad, en el plan de estudios existen dos espacios académicos relacionados: «Taller de creación de empresas»y «Formulación de proyectos turísticos». Desde estos se brindan las herramientas metodológicas para el desarrollo de ideas de emprendimiento que en el momento de materializarse se convierten en opción de grado.

\subsubsection{Administración ambiental.}

Subraya las características o cualidades de las personas que tienen el poder de visualizar oportunidades de negocios, lo que genera soluciones puntuales a necesidades urgentes de una población y establece así las actividades necesarias para planificar y ejecutar acciones en beneficio de la puesta en marcha de las unidades productivas.

\subsubsection{Ingeniería civil.}

Se reconoce como una iniciativa bajo la cual una persona desarrolla un proyecto con metas específicas que le permitan lograr objetivos a mediano y largo plazo, asumiendo un riesgo económico con el fin de aprovechar oportunidades del medio o del mercado. El emprendedor crea oportunidades donde otros no las ven. La experiencia en el tema nunca se ha proyectado más allá del aula de clase, de modo que corresponde al rol del estudiante quien, eventualmente, desarrolla productos o proyectos en función de ideas emprendedoras y más en función de lograr objetivos académicos.

\subsection{Desde los estudiantes}

Para el abordaje de las percepciones de este grupo se definió como población total a los estudiantes de los cursos III y IV, de todos los programas, por considerarse que serían los primeros en entrar a procesos de formación en el tema de emprendimiento a partir del 2018.

Se identificaron trescientos cuarenta y dos estudiantes con pesos relativos especificados en la tabla 2. De ahí se observan ciento tres estudiantes, de los cuales se encuestaron cuarenta y tres mujeres y sesenta hombres, en cada curso, al azar. Ingeniería civil corresponde al de mayor peso relativo con el 37,9\%, y Administración turística a la menor participación, con el 4,9\%. Entre Contaduría pública, Ingeniería financiera e Ingeniería de sistemas suman $40,7 \%$ como peso relativo, con lo cual se confirma la importancia de la muestra para el estudio.

Tabla 2. Muestra percepción estudiantes

\begin{tabular}{ccccccc}
\hline & Población IV + III & \% & N. ${ }^{\mathbf{0}}$ encuestas & Peso relativo & F & M \\
\hline Ambiental & 32 & 9,4 & 10 & 9,7 & 7 & 3 \\
Logística & 23 & 6,7 & 7 & 6,8 & 3 & 4 \\
Turística & 20 & 5,8 & 5 & 4,9 & 4 & 1 \\
Sistemas & 63 & 18,4 & 19 & 18,4 & 3 & 16 \\
Contaduría & 43 & 12,6 & 13 & 12,6 & 8 & 5 \\
Financiera & 34 & 9,9 & 10 & 9,7 & 3 & 7 \\
Civil & 127 & 37,1 & 39 & 37,9 & 15 & 24 \\
\hline Total & $\mathbf{3 4 2}$ & $\mathbf{1 0 0 , 0}$ & $\mathbf{1 0 3}$ & $\mathbf{1 0 0 , 0}$ & $\mathbf{4 3}$ & $\mathbf{6 0}$ \\
\hline
\end{tabular}

Fuente. Elaboración propia.

En la tabla 3 se presenta el análisis de percepción de los estudiantes. 
Tabla 3. Percepciones desde los estudiantes

\begin{tabular}{cccccc}
\hline Concepto & Crea & Formaliza & Consolida & Todas & Ninguna \\
\hline Participación & 11,7 & 9,7 & 15,5 & 61,2 & 1,9 \\
\hline Experiencia & Familia & Propia & Alguien & Academia & Otro \\
\hline Participación & 22,3 & 5,8 & 14,6 & 56,3 & 1 \\
\hline Opción T.G. & Muy alta & Alta & Media & Baja & Muy baja \\
\hline Participación & 35,9 & 44,7 & 19,4 & 0 & 0 \\
\hline Formación & Muy alta & Alta & Media & Baja & Muy baja \\
\hline Participación & 42,7 & 46,6 & 9,7 & 1 & 0 \\
\hline
\end{tabular}

Fuente. Elaboración propia.

\subsection{Desde el conocimiento del concepto}

A nivel general se evidenció, tal como lo registra la tabla 3 , que el $61,2 \%$ de los estudiantes tienen claro el concepto desde la creación, la formulación y la consolidación de las empresas en el marco de los procesos de emprendimiento. Por otra parte, el $11,7 \%$ considera la creación como lo esencial, el $9,7 \%$ lo ve como formalización y el $15,5 \%$ como consolidación; solo el 1,9\%, literalmente, desconoce los procesos emprendedores, lo que es explicable ante la ausencia del desarrollo del área en programas como, por ejemplo, Ingeniería civil.

\subsection{Desde la experiencia}

Desde la experiencia, a nivel general, se encontró que los estudiantes guardan un referente familiar en esta actividad en un $22,3 \%$; por experiencia propia en el 5,8 $\%$; en otra persona en el 14,6 \%; y con dirección desde la academia en el $56,3 \%$. Se destaca el efecto que se puede lograr desde la función sustantiva de la docencia.

\subsection{Desde la opción de grado}

$\mathrm{Al}$ indagar acerca de la tendencia a que el emprendimiento en la seccional se convierta en una opción de grado, el grupo estudiantil componente de la muestra refleja un nivel alto — 44,7 \%—, y muy alto — 35,9 $\%$-, lo cual suma un 80,6\%. Esta es una indicación diciente de un marcado interés por parte de los estudiantes en esta nueva opción para graduarse, a la vez que alerta a la responsabilidad de la universidad a fin de que responda a esta importante expectativa.
Cabe anotar que la población restante muestra un nivel de interés medio, es decir, nadie considera la opción en un grado bajo y menos aún muy bajo.

\subsection{Desde la necesidad de formación}

$\mathrm{Al}$ indagar acerca del interés por recibir formación para el emprendimiento en la seccional, el 42,7\% refleja una clara inclinación, el 46,6\% tiene alto interés y la media es del 9,7\%. Esto significa que el 89 \% está muy dispuesto a recibir formación sobre el tema, con lo cual se confirman la disposición y la necesidad de que el emprendimiento en la seccional adquiera mayor relevancia.

Puede observarse que todos los programas manifiestan gran aceptación por recibir formación en emprendimiento, de modo que se circunscribe la diferencia en cifras al número de integrantes. Sin embargo, las ingenierías civil y de sistemas presentan los mayores porcentajes. Al sumar los niveles alto y muy alto estos llegan al $75 \%$ y $36 \%$, respectivamente. Por su parte, las administraciones, y los programas de Contaduría pública e Ingeniería financiera muestran un alto interés por la formación en el tema de emprendimiento.

A manera de síntesis, el emprendimiento no debe supeditarse a un programa académico, sino estar asociado a la institución, y, aunque debe operar como una unidad para realizar acciones coordinadas a través de ella, cada programa debe desarrollar una estrategia a fin de contribuir a las políticas, así como al plan estratégico y operativo de orden institucional. 


\section{La propuesta}

$\mathrm{E}^{\mathrm{n}}$ coherencia con los planteamientos de Veciana (1999), Cooper (2003), Low (2001) y Bruyat-Julien (2000), como se citan en Matiz (2009), dado su carácter novedoso y el hecho de estar aún en proceso de consolidación en el marco de la investigación, parece no existir unidad conceptual acerca del emprendimiento o entrepreneurship, ni de su objeto de estudio. Esto ha conducido a un enrarecimiento y una permeabilidad que suscita su confusión con algunas disciplinas.

Así las cosas, esta investigación pretende concretar los principales aspectos que soportan el emprendimiento como proyecto educativo para los estudiantes universitarios, así como plantea un programa educativo de formación-acción-creación-sostenibilidad, con el fin de resolver problemas, encontrar oportunidades y generar actividades de emprendimiento sostenibles en un contexto social, laboral y profesional que posibilite mejores condiciones de vida y mayor desarrollo regional.

La Propuesta de educación para el emprendimiento está dirigida a todos los estudiantes de la Universidad Piloto de Colombia, de la Seccional del Alto Magdalena, que de forma voluntaria quieran formarse, adquirir las competencias necesarias y desarrollar sus capacidades como personas emprendedoras, así como generar y concretar ideas de negocios orientadas al emprendimiento como aporte al desarrollo personal, social y económico del país. Se espera que esta propuesta forme a los futuros profesionales como emprendedores y creadores de empresas innovadoras exitosas desde principios de integralidad y en armonía con lo expuesto en la misión institucional:

Con conocimiento científico y crítico, con mentalidad abierta a todos los aspectos de la vida y del desarrollo nacional, considerados dentro de una visión global y en particular, de los que caracterizan el contexto de la comunidad colombiana; mediante la investigación científica y la formación integral del hombre. (Universidad Piloto de Colombia, 2009, p. 4)

\subsection{El concepto}

El concepto que, principalmente, fundamenta este estudio, corresponde al de entrepeneurship, el cual proviene del francés entrepeneur, cuya traducción al castellano corresponde a «emprendedor». Según la Real Academia Española (1791), como se cita en Pereira (2007, p.14), es «la persona que emprende o se determina a hacer y ejecutar con resolución y empeño alguna operación considerable y ardua», lo que, de acuerdo con el mismo autor, conlleva a diferenciar entre empresario y emprendedor, por cuanto el primero se refiere a quien «toma a su cargo alguna empresa o negociación», mientras que el emprendedor se caracteriza, en esencial, por la innovación permanente y continua, por su ser social y sus dimensiones culturales, económicas y psicológicas, desde el punto de vista sistémico y humanista.

Así las cosas, hoy por hoy reviste importancia el análisis del espíritu emprendedor ubicado en el área del conocimiento de la disciplina administrativa, sin perder de vista que la concepción monodisciplinar se queda corta, por lo que es necesario dar paso a relaciones y conceptuaciones multidisciplinares. En otras palabras, el concepto no solo evoca la oportunidad, ni cómo es la persona, ni las capacidades organizacionales de cada uno, sino todo lo contrario: es un espacio de relaciones entre cada una de estas condiciones cada vez más complejas, de modo que se llega a considerar que las dimensiones contextuales, temporales y sociales confirman la interpretación del emprendedor como tal.

Por esto se modela al emprendedor piloto como generador de ideas, constructor permanente, aprendiz en continuo desarrollo de competencias, metas y objetivos, quien efectúa transformaciones y vuelve sobre el proceso a fin de seguir innovando. Esta es la base para construir una educación que fortalezca los factores de éxito identificados en sus variaciones particulares. 
Además, se advierte el siguiente interrogante: ¿El emprendedor transfiere conocimiento? Es decir, en un proceso colaborativo al interior de la institución, ¿desarrolla saberes generados en la actividad investigativa — que es el ámbito en el que surge nuevo conocimiento- con el objetivo de lograr que la comunidad académica pueda aplicarlo a sus finalidades en el entorno particular de cada programa académico?

Cabe aclarar que los investigadores de la Universidad Piloto de Colombia, estudiantes y docentes, participan en la transferencia del conocimiento en las tres funciones sustantivas desde la actividad de enseñanza — aprendizaje en procesos de formación, en el trabajo con los semilleros de investigación, en el ámbito de la investigación formativa y en los trabajos de grado-; a través del desarrollo propio de la docencia, con la investigación disciplinar que es transversal a toda la malla académica; y en la generación de conocimiento sustentado en la pertinencia, la relevancia y la sustentabilidad de los procesos que se llevan a la sociedad en cuanto a proyección e impacto.

\subsection{El modelo}

Además de la concepción propuesta sobre el emprendedor y la contemplación de algunos factores de éxito que, lógicamente, están ubicados en las acciones del espíritu empresarial —intenciones, pensamientos, expectativas, potencialidades, limitaciones del entorno particular, actitud positiva, etc.-, se exige determinar los componentes a desarrollar en el proceso educativo, lo cual, básicamente, se refiere a considerar las relaciones entre las variables definidas, para lo cual se realiza un análisis sistémico entre estas en el marco del proceso educativo originado en el modelo propuesto (Osorio y Pereira, 2011):

- Educación para el emprendimiento: orienta, guía y dirige a los estudiantes a realizar acciones de emprendimiento y a ser actores sociales activos y protagonistas para su entorno.
- Acción emprendedora: crea, transforma y reconstruye el entorno con base en la acción del emprendedor para la generación de valor.

- Formación emprendedora: permite el desarrollo de competencias y habilidades a fin de que el estudiante sea capaz de actuar para el emprendimiento.

\subsection{Variables}

De acuerdo con la concepción del emprendedor propuesta y la alusión a algunos factores de éxito que, lógicamente, se ubican en las acciones del espíritu empresarial que se reconocen en sus intenciones, pensamientos y expectativas, así como las potencialidades y limitaciones de su entorno particular — las cuales están por encima dela limitacionesinherentes a pensar en solo tener una actitud positiva al emprendimiento, o a crear nuevas empresas, o a considerar las pequeñas empresas como el objetivo particular- se exige determinar los componentes a desarrollar en el proceso educativo. En este orden de ideas, siguiendo la ruta de autores como Pereira (2007), entre otros, se proponen las siguientes variables de discusión: a) el emprendedor realiza acciones emprendedoras; b) la acción emprendedora recibe el accionar del emprendedor; c) el entorno y aspectos cercanos o lejanos que afectan en las condiciones psicológicas, culturales y económicas; y d) la formación concreta en las relaciones entre el emprendedor y el entorno en el accionar de este como emprendedor.

\subsection{Emprendimiento dinámico innovador}

El análisis continúa a partir de considerar el centro de gravedad de la propuesta para la seccional como un emprendimiento dinámico innovador, el Centro de Emprendimiento y la estructura de articulación del emprendimiento en el marco de los trabajos de grado y, por último, el desarrollo de los aspectos relacionados con los momentos o etapas del emprendimiento incubación, aceleración y formalización-.

El emprendimiento dinámico innovador se entiende como un proceso cuyos horizontes están de acuerdo 
con la visión de la universidad en cuanto a metas pertinentes, replicables y sostenibles, de alto impacto con relación a sus productos y servicios y, en general, a los modelos de negocio. Es decir, un emprendimiento con metas agresivas, de retos y de alto impacto (García, Rodriguez, Schnarch y Vesga, 2015).

El emprendimiento dinámico innovador implica procesos que, evidentemente, generan empleo y potencialización en la consecución de riqueza, pero, sobre todo, rompen la barrera en lo que se refiere a las posibilidades de incrementos en la productividad vía productos, servicios y modelos de negocios innovadores, esquemas de indagación y descubrimientos con métodos aplicables en diversos sectores y radios de acción. Es un emprendimiento ligado a la investigación que propende a su pertinencia en la región y el país, y por su replicabilidad en el aula y en la práctica empresarial; tiene, además, un carácter sostenible en el tiempo y el espacio, así como guarda, como lo plantea la misión institucional, respeto por el medio ambiente.

Por esta vía, las ideas serán empresas en potencia, de modo que reordenan la concepción de modelos de negocios orientados a las satisfacciones de necesidades y gustos de los clientes, pero, sobre todo, serán la base para el crecimiento económico y social de la región y el país.

García et al. (2015) caracterizan a este tipo de emprendimiento como «la vocación de rápido crecimiento, la diferenciación a través de la innovación y la ambición y la capacidad de emprender para asumir riegos». De esta manera, se hace específica la necesidad de que la universidad asuma también el reto de propiciar los espacios académicos, pedagógicos e investigativos pertinentes para que se aprovechen las condiciones particulares de estudiantes y docentes en cuanto a su creatividad, dinámica y competencias propias.

\subsubsection{Etapas de desarrollo del emprendimiento dinámico innovador.}

No existe consenso absoluto y preciso acerca de las etapas de un emprendimiento, ni en su duración ni en su caracterización. Sin embargo, se opta por retomar la clasificación elaborada por García et al. (2015), la cual ha sido adaptada para el presente estudio de la siguiente manera:

- Emprendedor piloto semilla: etapa de descubrimiento e ideas iniciales en la que los emprendedores desarrollan su proyecto, pero aún no lo logran consolidar frente a las necesidades y condiciones de los posibles compradores - consumidores-mercado-. El objetivo principal de esta etapa es articular la idea de producto y o servicio frente a las necesidades potenciales de los consumidores en el marco de las ventas.

- Emprendedor piloto incubador: se trata del crecimiento inicial, en el cual los estudiantes emprendedores tienen ideas claras y desarrollan el bien o servicio que debe tener demanda en el mercado; por tanto, generan los primeros ingresos. El objetivo es consolidar los procesos de adquisición de clientes y productos.

- Emprendedor piloto acelerador: las características y la tipificación de los productos, bienes y o servicios se encuentran plenamente identificados y cuentan con una demanda ya específica. El objetivo es desarrollar la demanda y conseguir un crecimiento permanente en ventas.

- Emprendedor piloto formalizador: el ritmo de incremento del mercado es importante, por lo que el objetivo es afianzar la productividad y las metas funcionales para la competitividad. Se debe cumplir con todos los requisitos comerciales y de ley a fin de iniciar procesos de diversificación de productos y modelos de negocios, es decir, dinámica.

\subsection{Proyectos de desarrollo empresarial}

Los proyectos desarrollados en los procesos de emprendimiento, aplicados al sector empresarial, se plantean como propuestas de creación de nuevas empresas mediante la articulación de la investigación como componente fundamental. 
Figura 1. Desarrollo del programa emprendimiento dinámico innovador

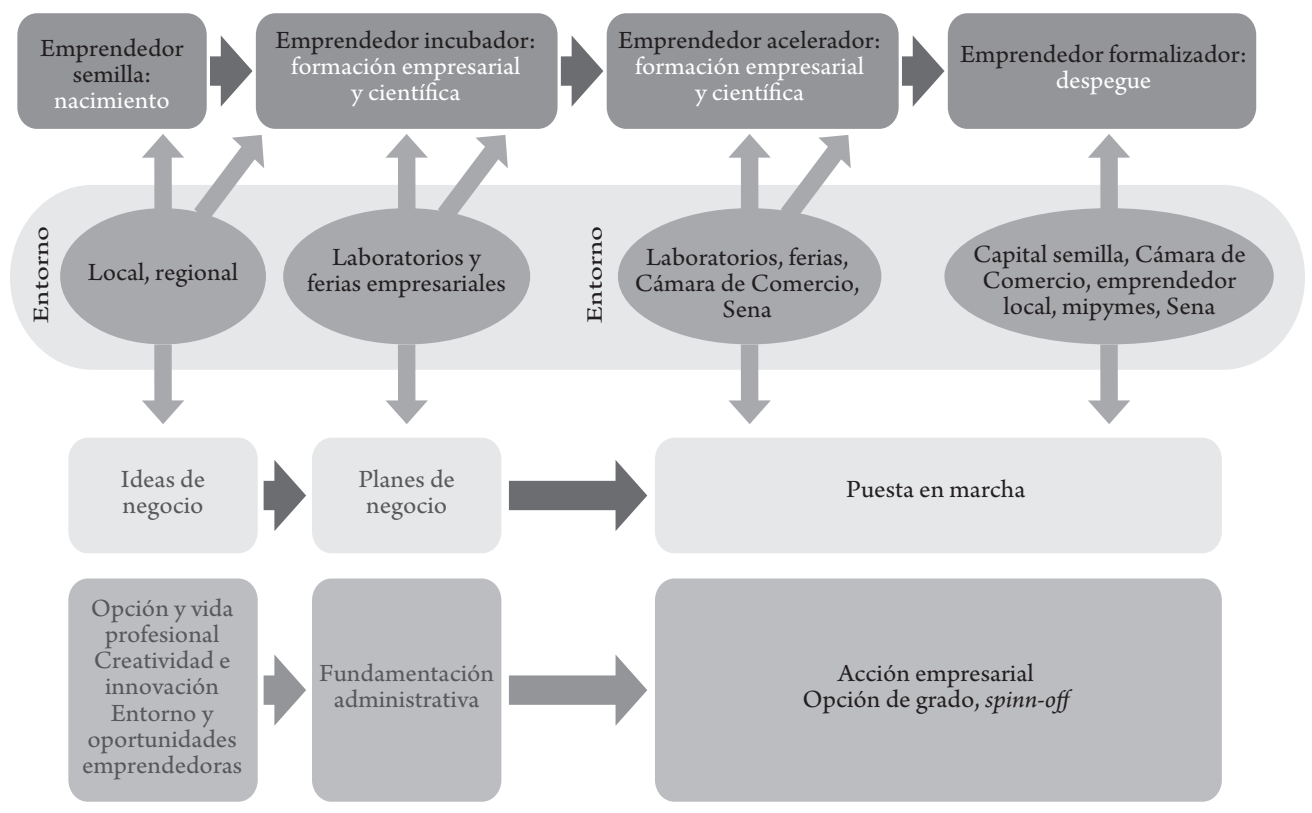

Fuente. Elaboración propia.

En la figura 1 se ilustra el proceso de desarrollo del emprendimiento dinámico, el cual, por lo general, pasa por los tres estados básicos: ideas de negocios, planes de negocios y puesta en marcha. Cada uno de estos estados tiene sus particularidades: en la idea de negocios se ubica el emprendedor semilla, con base en el entorno local, regional y con estudiantes, en busca de oportunidades emprendedoras; en los planes de negocios, básicamente se ubica la incubación con formación empresarial y científica - toda la fundamentación administrativa y los planes de negocios, la cual continúa con el proceso de aceleración en escenarios empresariales e institucionales, como, por ejemplo, el Centro de Emprendimiento, el SENA, la Cámara de Comercio, etc.-; y, finalmente, la puesta en marcha que se concreta en la formalización y el despegue de la empresa, valga decir, la acción empresarial, para lo cual son productos propicios las opciones de grado y las spin off.

Por último, la empresa puesta en marcha se concibe como una empresa constituida legalmente, con el cumplimiento de los trámites establecidos por el gobierno colombiano o en el curso de estos. Una empresa será puesta en marcha cuando el plan de negocios sea viable.

\subsection{Centro de Emprendimiento}

El emprendedor es la persona o ente que enfrenta con resolución situaciones y acciones difíciles y quien, frente a estas, está dispuesto a asumir el riesgo económico necesario para afrontarlo. Bajo esta consideración, dada su naturaleza el emprendedor bien puede ser un estudiante, un egresado, un docente o bien un administrativo de la universidad, un empresario o una empresa con iniciativa o necesidades de innovación o emprendimiento.

Por esto, el Centro de Emprendimiento se convierte en un gran reto y esfuerzo para la Universidad Piloto de Colombia, en su seccional del Alto Magdalena, en el propósito de dirigir su mirada educativa hacia una visión de fuertes desarrollos que promuevan la generación de grandes valores agregados con la autonomía de la persona o el ente emprendedor que actúa y se genera su propio trabajo, así como sus medios de sostenimiento. 
En la figura 2 se ilustra el proceso del Centro de Emprendimiento, el cual lo compone un equipo interdisciplinario que realiza apoyo, asesoría y consultoría a procesos de emprendimiento, desde la idea hasta la consolidación de planes de negocios.
Se inicia con la sensibilización, luego sigue la capacitación y la identificación, y se culmina con la creación de planes para la concreción de empresas nuevas cuyo fin es el crecimiento y el desarrollo social en un marco de innovación para la competitividad.

Figura 2. Esquema funcional

\begin{tabular}{|c|c|c|c|c|}
\hline $\begin{array}{l}\text { Fomento a la } \\
\text { cultura } \\
\text { emprendedora }\end{array}$ & $\begin{array}{c}\text { Identificación } \\
\text { de ideas } \\
\text { innovadoras }\end{array}$ & $\begin{array}{c}\text { Formulación de } \\
\text { proyectos }\end{array}$ & $\begin{array}{c}\text { Puesta en marcha } \\
\text { y operación }\end{array}$ & $\begin{array}{l}\text { Consolidación y } \\
\text { fortalecimiento }\end{array}$ \\
\hline
\end{tabular}

Fuente. Elaboración propia.

\subsection{Incubadora de ideas de negocios}

Efectivamente, un momento clave en el emprendimiento es el crecimiento de las empresas. Sin embargo, esto proviene de que, desde el inicio, exista una búsqueda de las herramientas que permitan un nacimiento fuerte y sostenible. Si bien las empresas parten de ideas novedosas, innovadoras $\mathrm{y}$, seguramente, muy estimulantes y sofisticadas, no se puede solo partir de esta mirada, sino que es necesario tener claros los horizontes y los entornos, así como aspectos tales como el mercado y las condiciones financieras, además de la estructura legal en la cual se va a desenvolver la nueva empresa.

No bastan, pues, las ideas excelentes si no existe una aproximación clara acerca del plan de negocios específico; de allí la importancia de las incubadoras de negocios como herramienta clave.
La figura 3 ilustra los procesos de incubación de negocios. Es necesario advertir que en todo el proceso se realiza apoyo, asesoría y tutoría. En este orden de ideas, el proceso presenta cuatro etapas:

- Inducción: idea de negocio, primera evaluación.

- Preincubación: orientación para el desarrollo de la empresa.

- Incubación: revisión y seguimiento al desarrollo de la empresa.

- Posincubación: esto para, después de estar en funcionamiento, realizar su seguimiento continuo.

Todo lo anterior conduce al nacimiento y la clarificación de las ideas, los objetivos y la caracterización de la empresa, la delineación del plan de negocios, las adecuaciones y los ajustes, el inicio de operaciones y la evaluación permanente del negocio.

Figura 3. Procesos en la incubación de negocios

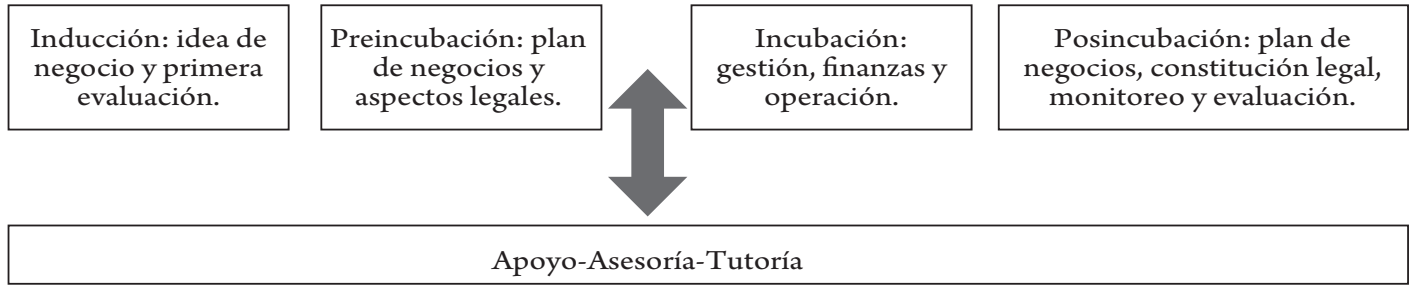

Fuente. Elaboración propia. 


\subsection{El concepto de aceleración empresarial}

En general, se puede afirmar que hace referencia a la velocidad con la que se desarrollan los procesos y las funciones de las organizaciones, así como a la forma como estos se concretan, siempre con el horizonte de los objetivos empresariales y las estrategias definidas para el negocio, dentro de un marco temporal definido.

Si bien es cierto que los emprendedores verdaderos, en el universo de la complejidad competitiva, son osados y arriesgan el todo por el todo, también lo es el hecho de que - hasta donde sea posiblese deben minimizar estos riesgos; es así que en la última década ha tenido lugar la aparición de los emprendedores sociales, quienes, sin perder la connotación de empresas sin ánimo de lucro, se dan a la tarea de impulsar la cooperación, de tal forma que la aventura resulte menos riesgosa.

En este orden de ideas, los emprendedores se encuentran ante una nueva tendencia de apoyo a las pymes, las cuales son estas sociedades u ONG que tienen como objetivo brindar apoyo empresarial a las empresas ya existentes o nacientes. Este es el papel que debe jugar la universidad, no solo en la formación de sus estudiantes como emprendedores, sino como fuentes de sustento para el crecimiento y la sostenibilidad de las empresas existentes y nacientes en el contexto del Alto Magdalena.

En el marco de los procesos de aceleración, por lo general, intervienen entidades externas a la organización, bien sean públicas o bien privadas, cuya misión es realizar el acompañamiento yla orientación en el desarrollo y la ejecución de las actividades y funciones establecidas a través de la transferencia de conocimientos, de las prácticas y los procesos, con la finalidad de conseguir el cumplimiento de las metas operativas y empresariales trazadas desde sus planes organizacionales.

La aceleración empresarial puede enfocarse en una o varias áreas de la organización: puede estar orientada al área financiera, comercial, de calidad, de mercadeo o de talento humano, pero siempre en busca de generar estrategias que fortalezcan las capacidades y los conocimientos de los equipos de trabajo.

Las aceleradoras son organizaciones que potencian el desarrollo de las empresas mediante acciones de innovación, consultoría y gestión, a fin de consolidarlas en los diferentes mercados locales, nacionales e internacionales mediante el logro de altos niveles de productividad.

Así, mientras las incubadoras se desenvuelven en el marco de las ideas a fin de lograr empresas de alto impacto, las aceleradoras, basadas en la innovación $\mathrm{y}$, primordialmente, con modelos muy competitivos, despliegan sus acciones a fin de potenciar el crecimiento permanente de las empresas en mercados de alto impacto.

\subsection{Formalización empresarial}

Otra función que se plantea el Centro de Emprendimiento es la participación activa y decisiva en los procesos de formalización empresarial.

El ejercicio de plantear, con la mayor objetividad, el concepto, la importancia y el porqué de la formalización de las empresas, implica una revisión teórica general en los principales entes que, de alguna manera, se encuentran comprometidos con el tema. Tal es el caso del Ministerio de Cultura Industria y Comercio, Impulsa Colombia, la Cámara de Comercio de Bogotá, Región Empresaria Colombia Emprende, el SENA y, de manera muy particular, la Ley 1429 de 2010 — la denominada Ley de Formalización y generación de empleo-. De allí surgen entonces las precisiones realizadas a la luz del desarrollo planteado por el mismo Estado, en la política nacional de emprendimiento, la Ley 1014 de 2006 del fomento a la cultura del emprendimiento.

Existe así una relación importante y significativa en cuanto al desarrollo de las políticas de crecimiento y desarrollo social a partir de la innovación para el emprendimiento bajo un estímulo permanente en la necesidad de la formalización empresarial.

Por esto, la tarea inicial es, precisamente, la de la 
formación de los empresarios en el tema de discernir y sopesar los beneficios y responsabilidades sociales que conlleva la formalización. Se puede decir, entre otros aspectos, que esta ley es favorable para la formalización, pues se encargó de disminuir los costos y obligar a las entidades respectivas a dar apoyo efectivo e integral para el desarrollo de las pequeñas empresas, todo ello al tener en cuenta la alta tasa de las que se cierran en los primeros años de estar constituidas.
En este orden de ideas, ante las preguntas «iqué se entiende por formalización?» y «icuáles son sus beneficios?», vale enfatizar lo siguiente. Una empresa está formalizada cuando dispone de las condiciones y las herramientas mínimas que requiere para su constitución, operación y funcionamiento, y cuando está en la vía de la legalidad, la ética, la seriedad, la responsabilidad social empresarial y la conservación y sostenibilidad del medio ambiente.

\section{Conclusiones}

$\mathrm{C}$ on base en las consideraciones teóricas conceptuales, la visión desde la investigación en emprendimiento, la revisión de las condiciones institucionales y el análisis de las relaciones entre variables en el tema de emprendimiento se elaboró la propuesta para la Universidad Piloto de Colombia, Un modelo piloto de emprendimiento dinámico e innovador, la cual se presenta a partir de un eje articulador denominado «Centro de Emprendimiento».

Se determinaron las variables que componen la línea de investigación, las cuales son: emprendedor quien realiza acciones de emprendimiento-, el acto mismo de emprender - que recibe precisamente el accionar del emprendedor-, el entorno - los aspectos o circunstancias que impactan en las condiciones sociales, económicas y sociales-, y, finalmente, las relaciones que se suceden entre el emprendedor y el entorno por las acciones de los actos de emprendimiento.

Se identificó el ámbito de las acciones emprendedoras para la línea, el cual se concreta en los sectores productivos y comerciales, en particular, con una gran influencia de base tecnológica para impulsar la innovación y el desarrollo. Allí se articulan los procesos con base en la transdisciplinariedad y en los diferentes programas académicos, de manera que se propicia una vocación de rápido crecimiento y diferenciación a través de la innovación y la capacidad de afrontar y asumir los riesgos que se sucedan en los procesos.

En este sentido, se identificó la ruta de la línea, la cual se estructura al nacer en las ideas, en los planes de negocios y en la puesta en marcha. Todo esto pasa por pilares básicos de semilla, incubación, aceleración y formalización.

En estos cuatro pilares se encuentra la ideación como origen básico del conocimiento emprendedor en el que se da espacio para la imaginación como elemento constructor, hasta la incubación y los instrumentos de la comprensión, pues esas ideas novedosas, innovadoras $y$, seguramente, muy estimulantes y sofisticadas, concretan y amplían sus horizontes y entornos, en conformidad con aspectos tales como el mercado y las condiciones financieras, al tener en cuenta que no solo es una excelente idea sino que se inicia la construcción de un plan de negocios específico:

- Aceleración -haciendo-. Impacta sobre el entorno, a partir de potencializar el desarrollo de las empresas mediante acciones de innovación, consultoría y gestión para su consolidación.

- Emprender formalizando -en sociedad, alianzas participación cooperación-. La empresa debe entonces disponer de las condiciones y herramientas mínimas que requiere para su constitución, operación y 
funcionamiento, y estar en la vía de la legalidad, la ética, la seriedad, la responsabilidad social empresarial y la conservación y sostenibilidad del medio ambiente.

Se evidencia que estas instancias se convierten en la base del desarrollo del modelo «Dinámico Emprendedor Piloto Sam». Estos cuatro pilares se estructuran en el marco institucional del modelo de emprendimiento de la seccional.

Al asumir la visión desde la investigación y la generación de conocimiento en los últimos años, se puede afirmar que el proceso de desarrollo del emprendimiento aún está en construcción, y que la propuesta educativa presentada participa de esto.

\section{Referencias}

Chavarro, D.; Orozco, L. (2008). Universidad y emprendimiento. Revista Hallazgos, 76, 65-97.

Crissien-Castillo, J. (2009). Investigando el entrepreneurship tras un marco teórico y su aporte al desarrollo económico de Colombia. Revista Escuela de Administración de Negocios, (66), 76-77. DOI: https://doi.org/10.21158/01208160. $\underline{\mathrm{n} 66.2009 .473}$

García, O.; Rodríguez, M.; Schnarch, D.; Vesga, R. (2015). Emprendedores en crecimiento. Bogotá: Editorial Kimpres. Recuperado de https://bit.ly/2IUSvHA

Garnsey, E. (2007). The entreprenurial university. The idea and its critics. En S Yusuf \& K. Nabeshima (Ed.). How Universities Promote Economic Growth (227-236). Washington: The World Bank.

Ley 1014 de 2006. (26 de enero de 2006). De fomento a la cultura del emprendimiento. Diario Oficial, núm. 46.164. Congreso de Colombia.
Esta cultura para el emprendimiento debe ser la base para la creación, la competitividad y la sostenibilidad de las empresas, con el apoyo del sector académico, los gremios y el Estado, con metas afines que se concreten en el desarrollo económico y social del país.

Así las cosas, resulta evidente la importancia de fortalecer la investigación en emprendimiento al mejorar sus niveles cuantitativos y cualitativos, así como al definir un derrotero o una argumentación real y significativa que permita soportar todo tipo de políticas y proyectos en apoyo al tema, sobre la base de la triada fundamental de desarrollo: universidad, empresa y Estado.

Matiz, F. J. (2009). Investigación en emprendimiento, un reto para la construcción de conocimiento. Revista Escuela de Administración de Negocios. (66), 169-182. DOI: https://doi. org/10.21158/01208160.n66.2009.480

Osorio, F.; Pereira, F. (2011). Hacia un modelo de educación para el emprendimiento: una mirada desde la teoría social cognitiva. Cuadernos de administración, 24(43), 13-33.

Pereira, F. (2007). La evolución del espíritu empresarial como campo del conocimiento. Hacia una visión sistémica y humanista. Cuadernos de Administración, 20(34), 11-37.

Universidad Piloto de Colombia. (2009). Proyecto educativo institucional. Bogotá: Universidad Piloto de Colombia. 
